\title{
Laser-Assisted Fabrication for Metal Halide Perovskite-2D Nanoconjugates: Control on the Nanocrystal Density and Morphology
}

\author{
Athanasia Kostopoulou ${ }^{1, *}$, Konstantinos Brintakis ${ }^{1}\left(\mathbb{D}\right.$, Efthymis Serpetzoglou ${ }^{1,2}$ and \\ Emmanuel Stratakis $1,2, *$ (D) \\ 1 Institute of Electronic Structure and Laser, Foundation for Research and Technology-Hellas, \\ 71110 Heraklion, Crete, Greece; kbrin@iesl.forth.gr (K.B.); eserpe@iesl.forth.gr (E.S.) \\ 2 Department of Physics, University of Crete, 71003 Heraklion, Crete, Greece \\ * Correspondence: akosto@iesl.forth.gr (A.K.); stratak@iesl.forth.gr (E.S.); \\ Tel.: +30-2810-391874 (A.K.); +30-2810-391274 (E.S.)
}

Received: 12 March 2020; Accepted: 9 April 2020; Published: 14 April 2020

check for updates

\begin{abstract}
We report on a facile and rapid photo-induced process to conjugate graphene-based materials with metal-halide perovskite nanocrystals. We show that a small number of laser pulses is sufficient to decorate the 2-dimensional (2D) flakes with metal-halide nanocrystals without affecting their primary morphology. At the same time, the density of anchored nanocrystals could be finely tuned by the number of irradiation pulses. This facile and rapid room temperature method provides unique opportunities for the design and development of perovskite-2D nanoconjugates, exhibiting synergetic functionality by combining nanocrystals of different morphologies and chemical phases with various $2 \mathrm{D}$ materials.
\end{abstract}

Keywords: 2D materials; laser-induced synthesis; anion exchange; nanoparticles; graphene oxide; photo-induced processes; synergistic effects

\section{Introduction}

Nanoconjugates consisting of nanocrystals coupled with graphene-based materials have recently attracted the interest of the scientific community for their synergistic properties originating from the coupling of the two different materials [1-7]. The functional groups (hydroxy, carbonyl, carboxy) on their basal planes or edges of the 2D materials-which make them soluble in multiple solvents, coupled with their low dimensionality - offer attractive properties for applications ranging from photovoltaics to photodetectors and sensors [8-11]. The coupling of these 2D materials with semiconducting quantum dots, mainly synthesized in colloidal solutions, explores the usage of these materials in light-harvesting [12], as catalysts for oxygen reduction in fuel cells [2], electrocatalysts for water splitting [5], as well as in Li-ion batteries [3] derived from the energy and charge transfer, [4,13] or synergetic and enhanced properties $[5,14]$ of the complex structures.

In recent years, the plethora of synthesis pathways for the fabrication of lead halide perovskite nanocrystals has given rise to their conjugation with 2D counterparts, in order to explore new synergetic phenomena [14-19]. The metal halide perovskite nanocrystals are a new class of nanomaterials; their properties can be modified by tuning the halide component [20], or by altering their dimensionality or morphology [21,22]. Anion exchange reactions can be used to change the optical properties of these nanostructures. The ions $\mathrm{Cl}^{-}$and $\mathrm{I}^{-}$can partially or totally substitute $\mathrm{Br}^{-}$in the crystal structure of the $\mathrm{CsPbBr}_{3}$ through a chemical anion exchange reaction in solution $[20,23]$ or by a photo-induced process in a $\mathrm{Cl}^{-}$or $\mathrm{I}^{-}$containing solvent [24]. Furthermore, in such thin nanocrystals, the optical properties may be tuned by modifying the thickness of them (nanoplatelets) $[25,26]$. 
Different morphologies of metal halide perovskite nanocrystals, such as nanospheres [15], nanocubes [1,16-18,27] or nanowires [14] have been grown on 2D materials and utilized for applications in light harvesting [14], for photocatalytic $\mathrm{CO}_{2}$ reduction [15,17], for visible-light photocatalyst for $\mathrm{H}_{2}$ evolution in aqueous HI solution [28] and in photoelectric detection [16]. The multiple applications of the perovskite-2D nanoconjugates lead the way to find easy, fast, solution-processable and controllable methods to fabricate such complex structures with attractive properties. It is important to note here that the above nanoconjugates are synthesized by hot-injection or re-precipitation chemical methods at high [15,16] or mild [17] temperatures in which the 2D materials are presented during the perovskite nanocrystal synthesis. These syntheses need complex apparatus such as Schleck line and give uncontrollable density and non-uniform nanocrystals on the 2D-materials. As a result, the properties of the perovskite-2D nanoconjugates could be varied from one batch to another.

This work uses a completely different approach to anchor previously fabricated metal-halide perovskite nanocrystals on graphene-based materials. A laser-assisted method was used for the first time for the fabrication of perovskite-2D nanoconjugates in colloids. It is shown that a few femtosecond pulses suffice to decorate the flakes with metal-halide perovskite nanocrystals without the initial nanocrystal morphology to be affected. The nanocrystals density can be finely controlled by the number of irradiation pulses. First, the nanocrystals are decorated at the periphery of the flakes (number of pulses <1000) and then are attached on the basal plane of them (number of pulses $>1000$ ). This facile and rapid room temperature method provides unique opportunities for the cost-effective and large-scale synthesis of fully controllable perovskite-2D nanoconjugates by combining nanocrystals of different morphologies and chemical phases together with multiple 2D materials.

\section{Materials and Methods}

\subsection{Preparation of the Perovskite-GO Nanoconjugate Colloid}

A published protocol from our group based on a precipitation colloidal method was used for the synthesis of the metal halide nanocrystals [29]. $0.4 \mathrm{mmol} \mathrm{PbBr}_{2}$ (trace metals basis, 99.999\%, Sigma-Aldrich Corporation, St. Louis, MO, USA) and 0.4 mmol CsBr (anhydrous, 99.999\%, Sigma-Aldrich Corporation, St. Louis, MO, USA) were dissolved in $10 \mathrm{~mL}$ of DMF (anhydrous, 99.8\%, Alfa-Aesar, Haverhill, MA, USA) and left under stirring for three hours in the protective atmosphere of a glovebox. Then, $1 \mathrm{~mL}$ of oleic acid (technical grade, 90\%, Alfa Aesar, Haverhill, MA, USA) and $0.5 \mathrm{~mL}$ oleylamine (approximate C18-content 80 - 90\%, ACROS Organics, Geel, Belgium) were added in the above solution. All the chemicals were used without further treatment, except oleic acid which was degassed for $1 \mathrm{~h}$ at $120^{\circ} \mathrm{C}$. Afterwards, $0.9 \mathrm{~mL}$ of the precursor solution was added rapidly in $10 \mathrm{~mL}$ of anhydrous toluene $(10 \mathrm{~mL})$ in a sealed vial which was placed in ice. The solution was left under vigorous stirring (1000 RPM) for 30 minutes. Subsequently, it was retained on the bench for a week. Then, perovskite nanocrystals were separated upon centrifugation at 1000 RPM for 5 min and finally re-dispersed in 1,2-dichlorobenzene (DCB, spectrophotometric grade, 99\%, SigmaAldrich Corporation, St. Louis, MO, USA). Then, this solution was mixed with the DCB-based solution of the graphene oxide to proceed with the photo-induced process. GO was prepared from graphite powder according to a modified Hummers' method [30].

\subsection{Photo-Induced Process for the Fabrication of the Perovskite-GO Nanoconjugates}

The laser setup for the fabrication of the nanoconjugates comprised an Yb:KGW ultrafast pulsed laser source, two mirrors and a convex lens of $20 \mathrm{~cm}$ focal length (Figure 1). The experiments were conducted at ambient conditions at room temperature. The laser source produced linearly polarized pulses with 170 femtosecond pulse duration and was adjusted to generate $513 \mathrm{~nm}$ laser wavelength at $60 \mathrm{kHz}$ repetition rate. The quartz cuvette with the solution was fixed at $5 \mathrm{~cm}$ out-of-focus distance for all the irradiations. The Gaussian spot diameter was $700 \mu \mathrm{m}$ at $1 / \mathrm{e}^{2}$, which was measured and analyzed by using a CCD camera. The laser fluence was stable for all the experiments at the value of 
$0.5 \mathrm{~mJ} / \mathrm{cm}^{2}$ while the number of the pulses was changing in a wide spectrum, starting from a single until 115.2 million pulses.
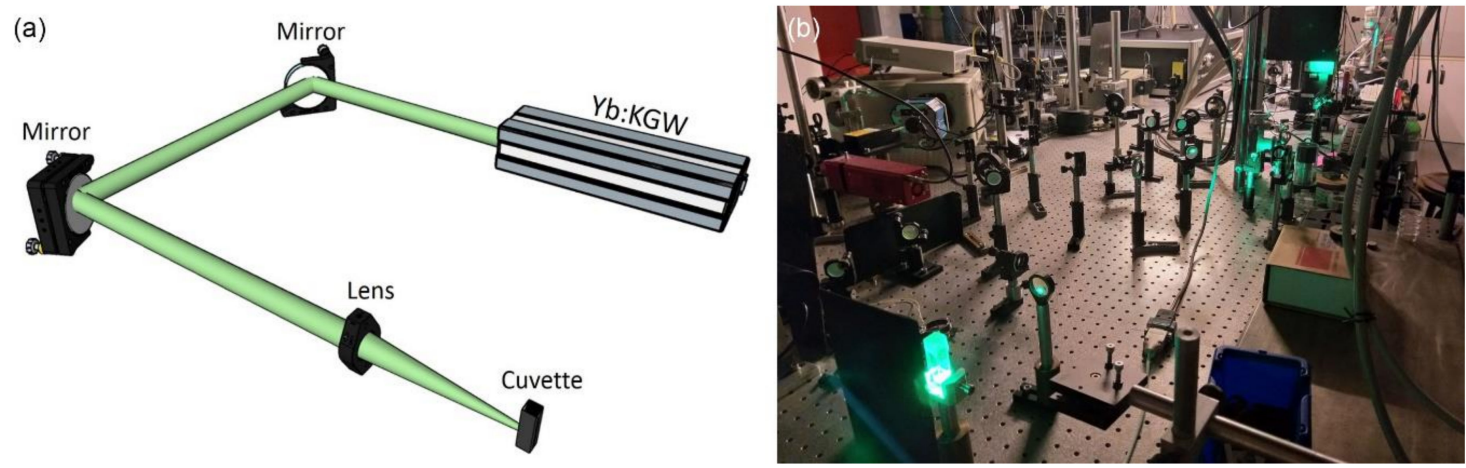

Figure 1. (a) Schematic representation and (b) photograph of the setup of the photo-induced process using a femtosecond laser.

\subsection{Characterization of the Materials}

Low magnification and HRTEM images were recorded on a LaB6 JEOL 2100 transmission electron microscope (JEOL Ltd, Akishima, Tokyo, Japan) operating at an accelerating voltage of $200 \mathrm{kV}$. All the images were recorded by the Gatan ORIUS TM SC 1000 CCD camera (Gatan Inc. Pleasanton, CA, USA). For the TEM analysis, a drop of the as-prepared and the irradiated DCB-based solution was deposited onto a carbon-coated copper TEM grid, which was eventually evaporated. The structural features of the nanocrystals were studied through FFT patterns obtained from the HRTEM images. About 130 individual nanohexagons were counted up for size distribution diagrams and for calculation of the nanocrystal mean size (diagonal length).

XRD studies were performed on a Rigaku D/MAX-2000H rotating anode diffractometer (Rigaku, Tokyo, Japan) with $\mathrm{Cu} \mathrm{K} \alpha$ radiation, equipped with a secondary graphite monochromator. The XRD data were collected at room temperature over a $2 \theta$ scattering range from $10^{\circ}$ to $40^{\circ}$, with a step of $0.02^{\circ}$ and a counting time of $20 \mathrm{~s}$ per step. The as-prepared and the irradiated samples were dried before the XRD experiments.

The UV-Vis absorption spectra of the solutions before and after laser irradiation were collected at room temperature on a Perkin Elmer, LAMBDA 950 UV/VIS/NIR spectrophotometer (PerkinElmer Inc., Waltham, MA, USA). The solutions were placed in quartz cuvettes.

The fluorescence emission of the solutions placed in quartz cuvette were measured at $300 \mathrm{~K}$ on a Fluoromax-P Phosphorimeter (Horiba Ltd., Kyoto, Japan) employing a $150 \mathrm{~W}$ Xenon continuous output ozone-free lamp.

Raman spectroscopy was performed on the GO solutions after evaporation of a small quantity using a Nicolet Almega XR Raman spectrometer (Thermo Fisher Scientific, Waltham, MA, USA) with a $473 \mathrm{~nm}$ blue laser as an excitation source.

\section{Results and Discussion}

A facile low temperature precipitation-based method was utilized for the synthesis of the metal halide perovskite nanocrystals. This synthesis protocol was published earlier from our group and these nanomaterials tested for their effectiveness as anode materials in $\mathrm{Li}$-air batteries utilizing aqueous electrolyte [29]. Metal halide nanohexagons of diagonal length $\mathrm{L}=93.5 \pm 11.5 \mathrm{~nm}$ were successfully synthesized, centrifuged and re-dispersed in dichlorobenzene (DCB). HRTEM images, together with the analysis of their respective FFT pattern of an individual nanohexagon, revealed the high-crystallinity of these nanocrystals (Figure 2a). The crystal structure of the nanohexagons was compatible with the rhombohedral $\mathrm{Cs}_{4} \mathrm{PbBr}_{6}$ crystal structure (ICSD-025124) (Figure 2a upper inset). Furthermore, weaker diffraction spots were observed in the FFT pattern which coincide with the (200) crystal planes 
(corresponding to lattice fringes with interplanar spacings of $0.4 \AA$ ) and with the (110) (interplanar spacings $5.4 \AA$ ) of the orthorhombic $\mathrm{CsPbBr}_{3}$ crystal structure (ICSD-97851). The coexistence of two phases of different crystal structure was also confirmed by the analysis of the XRD patterns (Figure 2b).
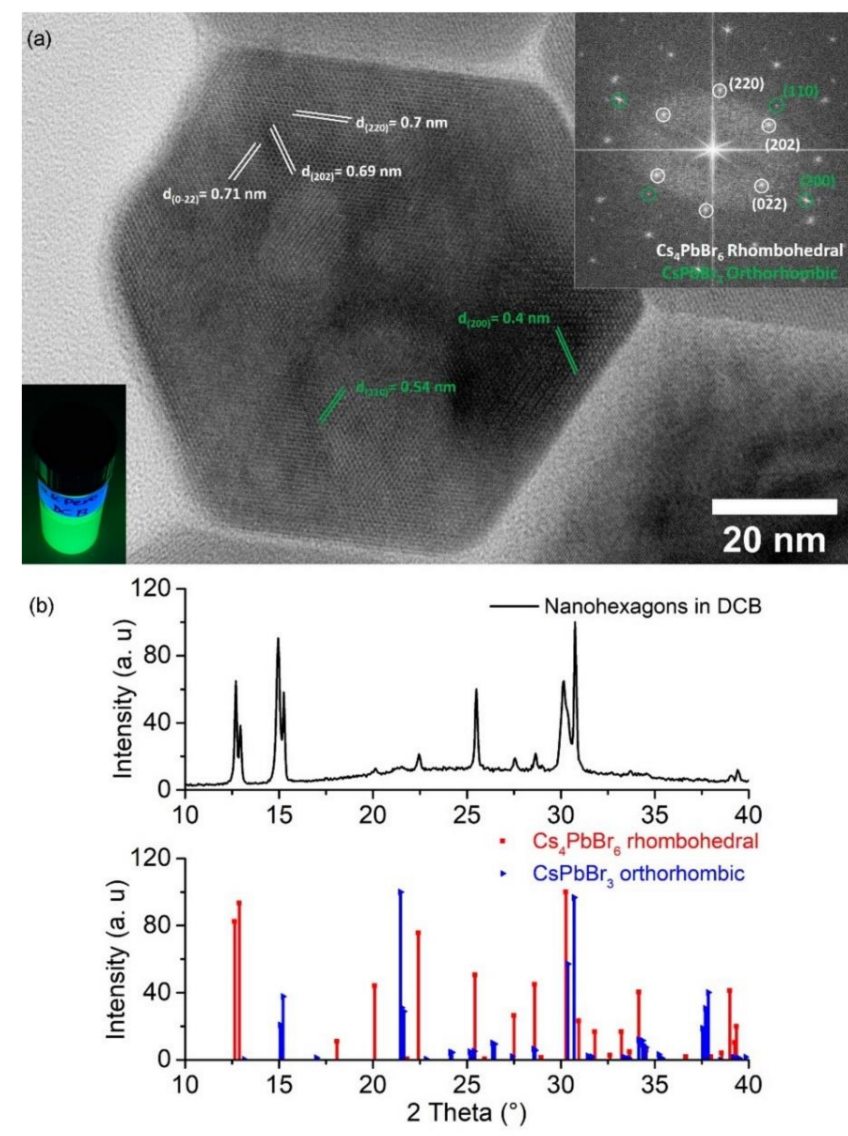

Figure 2. (a) High resolution TEM (HRTEM) image of the all-inorganic metal halide nanohexagons dispersed in dichlorobenzene. Insets: The fast Fourier transform (FFT) pattern of the HRTEM image of the individual nanohexagon (upper image) and colloidal solution photograph (under UV illumination, $\lambda=365 \mathrm{~nm}$ ) (bottom image); (b) XRD pattern of the same sample. The reference patterns of the rhombohedral $\mathrm{Cs}_{4} \mathrm{PbBr}_{6}$ crystal structure (ICSD-025124) and the orthorhombic (ICSD-97851) are also provided for comparison (red and blue pattern in $b$ ).

The solution of the nanohexagons was PL-active under UV illumination (Figure 2a, bottom inset). The origin of the bright green emission in $\mathrm{Cs}_{4} \mathrm{PbBr}_{6}$ nanocrystals is still under debate [31]. Two explanations have been given in the literature for this origin. In the first, the green emission was originated from the coexistence of two phases upon synthesis $\left(\mathrm{CsPbBr}_{3}\right.$ and $\left.\mathrm{Cs}_{4} \mathrm{PbBr}_{6}\right)$ [32]. In the second explanation, this emission was attributed to point-defects emission originating from the intrinsic features or bromide vacancies [33] in the non-fluorescent 0D perovskite structure [34]. Our indication is the first experimental observation that the strong photoluminescence of the $\mathrm{Cs}_{4} \mathrm{PbBr}_{6}$ nanohexagons arises from the inclusion of a fluorescent phase in a non-fluorescent similar to that observed in green emitting $\mathrm{Cs}_{4} \mathrm{PbBr}_{6}$ powders [35] or due to synergetic effects at $\mathrm{CsPbBr}_{3}-\mathrm{Cs}_{4} \mathrm{PbBr}_{6}$ interfaces [36].

Graphene oxide (GO) was also exfoliated in the same solvent (Figure 3a). The dichlorobenzene solvent was adopted to enable effective exfoliation of the GO with no effect on the perovskite nanocrystals. The nanocrystals were well-dispersed in the same solvent (Figure 3b). N,N-dimethylformamide (DMF) was tested also as dispersive solvent due to its appropriateness for exfoliation, but this had a strong influence on the perovskite nanocrystals (Figure S1). As a consequence, the DMF was not a good 
solvent for the fabrication of the nanoconjugates. The DCB-based solutions of the GO and of the metal halide perovskite nanocrystals were mixed and placed in a quartz cuvette.
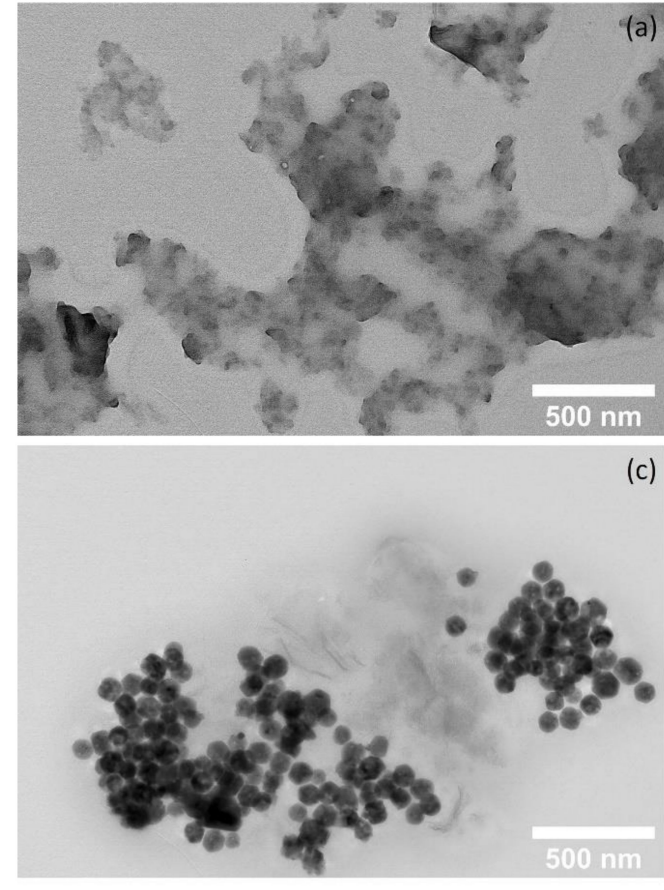
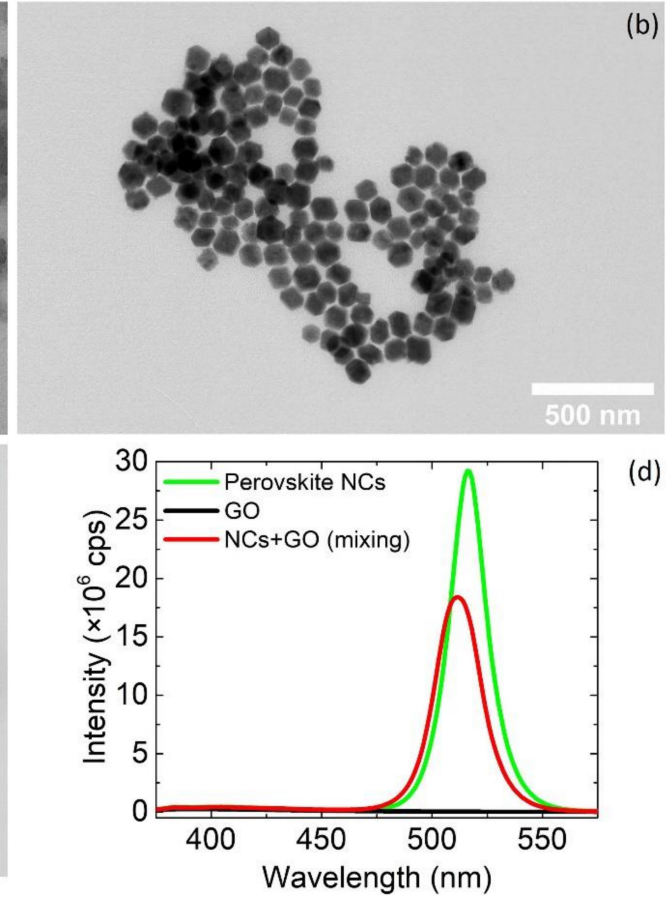

(d)

Figure 3. (a-c) TEM images of the exfoliated graphene oxide (GO) in 1,2-dichlorobenzene (DCB), of the metal halide nanocrystals in the same solvent and the mixture of the two before the laser irradiation; (d) Photoluminescence spectra of the same solutions.

Some of the nanocrystals were attached on the periphery of the GO flakes (Figure 3c) and most of them remain individually in the solution when the two solutions were mixed (Figure S2). The partial attachment of the nanocrystals on the GO flakes was also confirmed by the photoluminescence (PL) spectra (Figure 3d). In the case of the individual nanocrystals, a single peak was observed and centered at $516 \mathrm{~nm}$ (Figure 3d, green curve). The narrow size distribution of the nanocrystals was indicated from the narrow PL full-width half maximum (FWHM). Furthermore, when the nanocrystals were anchored on GO, the peak was blue shifted for only $4 \mathrm{~nm}$, but the intensity was decreased significantly (Figure $3 \mathrm{~d}$, red curve), which was also observed in similar nanoconjugates synthesized chemically $[15,16,28]$. Both solutions have strong and narrow absorption peaks at 311-315 $\mathrm{nm}$ and an absorption onset at $512 \mathrm{~nm}$ (Figure S3). This absorption features were in agreement with previous reports on nanocrystals of $\mathrm{Cs}_{4} \mathrm{PbBr}_{6}$ with the presence of a secondary $\mathrm{CsPbBr}_{3}$ chemical phase $[37,38]$. Furthermore, the sharp dip at around $330 \mathrm{~nm}$ has been observed in systems where these phases coexist [38,39]. These main features remain when the perovskite solution was mixed with the GO solution.

Then, a photo-triggered process was utilized to see if nanoconjugates with higher density of nanocrystals decorated on GO flakes could be obtained. A high repetition rate femtosecond laser system using a directly diode-pumped $\mathrm{Yb}: \mathrm{KGW}$ (ytterbium doped potassium gadolinium tungstate) as active medium, was employed for the irradiation of the mixed perovskite-GO solution (Figure 1). The laser wavelength, the repetition rate and the pulse duration used for that purpose were $513 \mathrm{~nm}$, $60 \mathrm{kHz}$ and $170 \mathrm{fsec}$, respectively. The laser fluence in all the experiments kept constant at the value of $0.5 \mathrm{~mJ} / \mathrm{cm}^{2}$ and only the number of the pulses was varied. The colloids obtained by this process, were stable and no precipitation was observed. The influence of the pulses number, ranging from 1 to $10^{6}$, on the morphologic characteristics of the nanoconjugates were examined by TEM (Figure 4a-g). The density of the nanocrystals was increased with the number of the pulses retaining their primary size (Figure S4) and the perovskite nanocrystals were assembled first on the periphery of the GO flakes and 
then on the basal plane when the number of the pulses was increased to over $10^{3}$ pulses. The peak of the PL of the irradiated solutions was located at the same wavelength with that of the simply mixing, but the intensity was reduced as the number of the pulses increases (Figure $4 \mathrm{~h}$ ). The same wavelength of the PL peak indicated that no anion exchange takes place for these irradiation conditions.

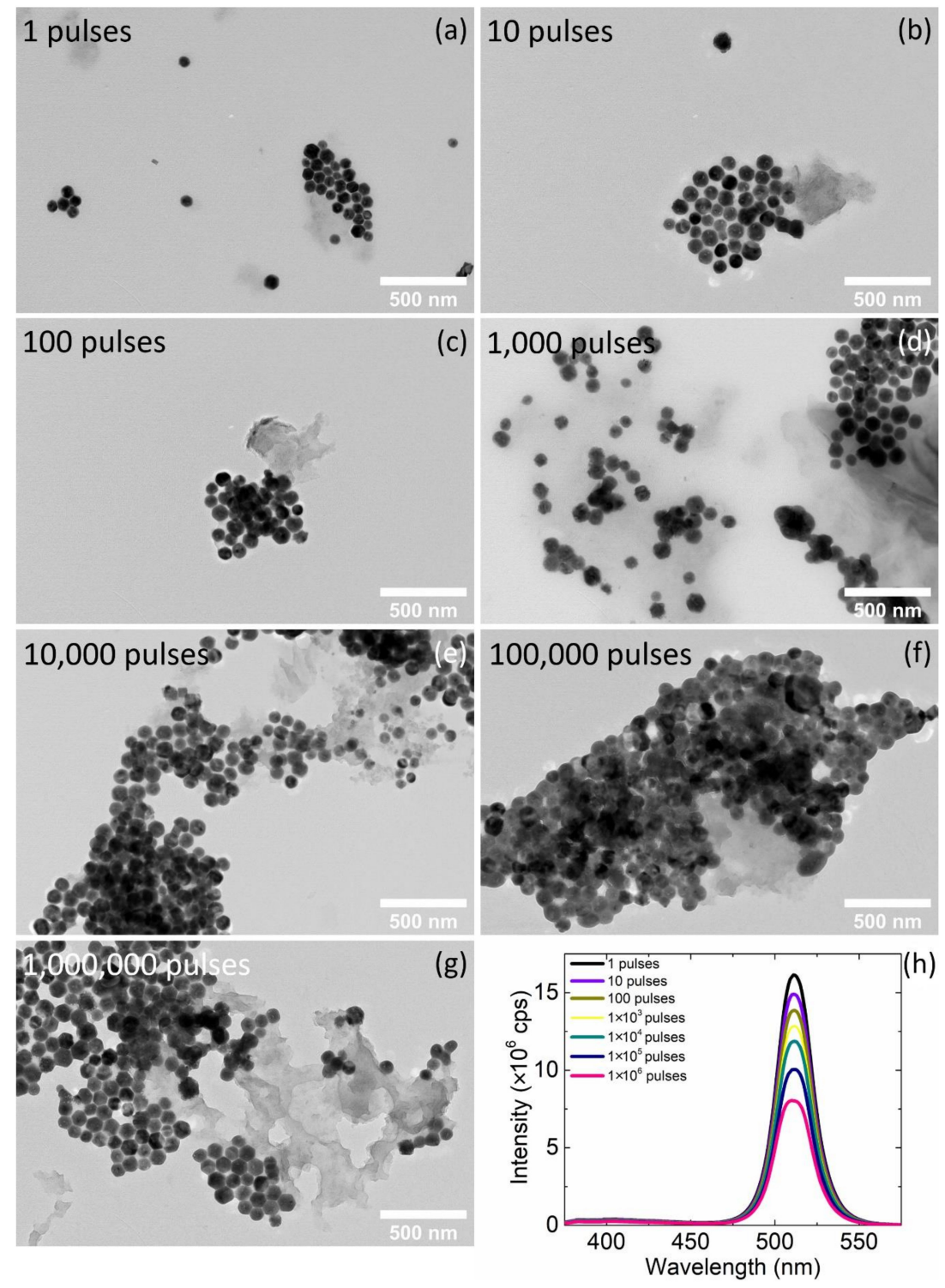

Figure 4. (a-g) TEM images of the perovskite-GO nanoconjugates after irradiation from 1 to $10^{6}$ femtosecond pulses; (h) Photoluminescence of the DCB-based irradiated solutions.

The scheme in the Figure 5 represents the proposed mechanism. The GO includes functional groups (hydroxyl, carbonyl, carboxylic) on their basal planes or edges which could be functional for nanocrystal anchor. Raman spectra indicated that no complete reduction of the as-prepared GO occurs in these irradiation conditions. The $\mathrm{D} / \mathrm{G}$ intensity ratio $\left(\mathrm{I}_{\mathrm{D}} / \mathrm{I}_{\mathrm{G}}\right)$ was 0.72 and 0.70 before and after laser-irradiation with 3.6 million pulses (Figure S5). This ratio decreases to 0.66 for even larger number of pulses (28.8 million pulses). According to previous reports, the carboxyl groups on the edges were coordinated mainly with $\mathrm{Pb}^{+2}$ ions of the metal halide nanocrystals crystal structure $[40,41]$.

The effect of the number of the irradiation pulses to the final morphology of the nanoconjugates was drawn from the careful observation of the TEM images (Figure 4 for 1 to $10^{6}$ irradiation pulses and Figure S6 for a larger number until $57.6 \times 10^{6}$ pulses). The perovskite nanocrystals remain on the 
periphery of the GO flakes for the irradiation duration from 1 to 100 pulses (Figure $4 a-c$ ) while for $10^{3}$ to $10^{4}$ pulses the density of the nanocrystals on the GO flakes increases significantly (Figure $4 \mathrm{~d}-\mathrm{f}$ ). For irradiation of the $10^{6}$ pulses, the nanocrystals were starting to detach from the basal planes of the flakes (Figure 4g, Figure S7a,b) and remain mainly around the periphery (Figure S7c,d) while in the case of $1.8 \times 10^{6}$ pulses the perovskite nanocrystals seem to start to be aggregated (Figure S8). From $14.4 \times 10^{6}$ pulses and longer irradiation duration, an anion exchange was started and thus two peaks were observed in the PL spectra (Figure S9). Similar behavior was observed for the same irradiation time, irradiating the perovskite nanocrystals without the GO (Figure S10). The initial peak remains at the same wavelength and decreases with the number of pulses while a new peak was emerged upon photo-triggered process in the absence of any reacting anion source [42]. The mechanism of anion exchange in Cl-containing solvent triggered by a laser has been proposed previously by Son's group [24]. Based on this mechanism, the anion exchange was realized via electron transfer from the perovskite nanocrystals to the solvent producing halide ions. Anion exchange of the 0D $\left(\mathrm{Cs}_{4} \mathrm{PbBr}_{6}\right)$ and $3 \mathrm{D}\left(\mathrm{CsPbBr}_{3}\right)$ phases, occurs at large number of pulses and could be confirmed from the careful analysis of the selected area diffraction patterns of the as-prepared and the irradiated samples (Figure S12). Acquiring the diffraction patterns from ensembles of mixed 3D/0D nanocrystals for the as-prepared nanocrystals (Figure S12a) and the $14.4 \times 10^{6}$ pulses irradiated samples (Figure S12b), one can spot differences between the two patterns. The $d$ spacings of the irradiated samples were decreased compared to the initial ones. They approach, but not reach the values for the chlorine $0 \mathrm{D}$ phase, indicating the partial anion exchange. In these irradiation conditions some of the nanohexagons remain on the GO, but the morphology of them start to modify and to become irregular (Figure $\mathrm{S} 11 \mathrm{a}, \mathrm{c})$. The perovskite nanocrystals start to aggregate, indicating desorption of the ligands from the particle surfaces (Figure S11b,d). At $57.6 \times 10^{6}$ pulses, nanocrystals of different shapes were observed (Figure S13). Some of the nanohexagons remain as previously but also quite spherical nanocrystals appeared from their partial fragmentation. The nanocrystals transform to nanoribbons through a laser-induced melting and subsequent recrystallization due to the laser energy absorption [43]. Very recently, similar structures have been observed through temperature-driven transformation and the rearrangement of atoms at the connecting facets [44].

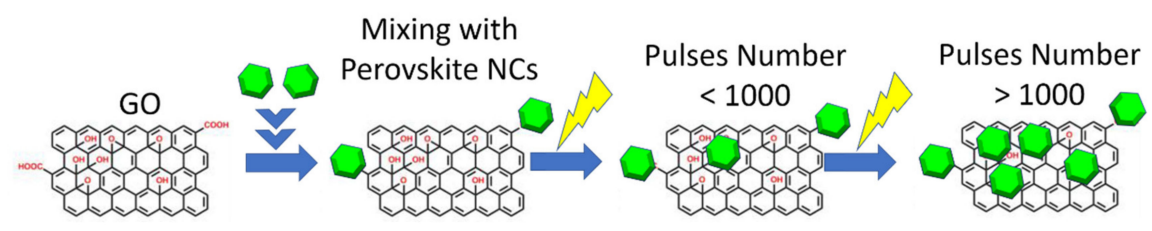

Figure 5. Proposed mechanism of the anchoring of metal nanocrystals on the GO flakes.

\section{Conclusions}

In summary, we have reported a facile laser-induced process to fabricate perovskite-2D nanoconjugates. In particular, pre-prepared nanocrystals were mixed with the GO materials in solution and irradiated with a femtosecond laser at ambient conditions. It was observed that the density of the nanocrystals on the flakes can be controlled by the number of the irradiation pulses. The laser pulses were efficient to promote the creation of functional groups on $\mathrm{GO}$ which allow perovskite crystals to bind to GO and cover the available flakes. The irradiation can damage perovskite crystals so the dose has to be kept sufficiently low ( $<1000$ pulses) to preserve their structure and morphology and eventually the luminescent properties. The solution of the nanoconjugates was PL-active following the irradiation process. The importance of this fast and easy process is that it presents a universal procedure to decorate 2D materials with metal halide nanocrystals. This laser-assisted process enables the creation of many conjugates with perovskite nanocrystals of various composition and shape. The nanoconjugates combine the exciting properties of the perovskite nanocrystals together with the interesting properties of the $2 \mathrm{D}$ materials. In addition, new physics and synergetic effects are 
emerging from the coupling between the two different materials. Among our future plans is to use this laser-assisted method to enhance the PL of the nanocomposites.

Supplementary Materials: The following are available online at http://www.mdpi.com/2079-4991/10/4/747/s1, Figure S1: TEM image of the irradiated DMF-based solution of GO with metal halide nanocrystals; Figure S2: TEM images of the mixture of the nanocrystals and the GO before the laser irradiation; Figure S3: Absorption spectra of the metal halide nanocrystals, the exfoliated GO and the mixture of the two before the laser irradiation in DCB; Figure S4: Size distribution diagrams for the nanohexagons before and after the laser irradiation; Figure S5: Raman spectra of the GO before and after 3.6 and 28.8 million pulses laser irradiation; Figure S6: TEM images and PL spectra for irradiation duration longer than $1 \times 10^{6}$ pulses; Figure S7: TEM images of the GO-perovskite composite after $10^{6}$ pulses irradiation; Figure S8: TEM images of the GO-perovskite composite after $1.8 \times 10^{6}$ pulses irradiation; Figure S9: PL spectra of the perovskite-GO composite solution after irradiation with 14.4, 28.8 and $57.6 \times 10^{6}$ pulses; Figure S10: PL spectra of the as-prepared nanohexagons solution after irradiation with 14.4, 28.8 and $57.6 \times 10^{6}$ pulses; Figure S11: TEM images of the GO-perovskite composite after $14.4 \times 10^{6}$ pulses irradiation; Figure S12: Selected area (electron) diffraction patterns of the as prepared nanocrystals and the $14.4 \times 10^{6}$ pulses irradiated samples; Figure S13: Different nanocrystal morphologies such as nanohexagons (a), nanoribbons (b) and quite spherical observed in the solution after irradiation with $57.6 \times 10^{6}$ pulses.

Author Contributions: Conceptualization, A.K. and K.B.; Methodology, A.K., K.B. and E.S. (Efthymis Serpetzoglou); Validation, A.K. and K.B.; Investigation, A.K. and K.B.; Resources, A.K.; Data curation, A.K., K.B.; Writing-Original draft preparation, A.K.; Writing-Review and editing, A.K., K.B. and E.S. (Emmanuel Stratakis); Visualization, A.K. and K.B.; Supervision, A.K. and E.S. (Emmanuel Stratakis); Project administration, A.K and E.S. (Emmanuel Stratakis); Funding acquisition, E.S. (Emmanuel Stratakis). All authors have read and agreed to the published version of the manuscript.

Funding: The research was co-financed by the Greece and European Union (European Social Fund-ESF) through the Operational Program, "Human Resources Development, Education and Lifelong Learning 2014-2020" in the context of the project, "Development of hybrid 2D-perovskites materials for enhanced efficiency of perovskite solar cells" (MIS 5004411).

Acknowledgments: The research was co-financed by the Greece and European Union (European Social Fund-ESF) through the Operational Program, "Human Resources Development, Education and Lifelong Learning 2014-2020" in the context of the project, "Development of hybrid 2D-perovskites materials for enhanced efficiency of perovskite solar cells" (MIS 5004411).We would like to thank Kyriaki Savva, Nikolaos Livakas and Michaila-Akathi Pantelaiou for their help in the laser experiments and Minas Stylianakis for the preparation of the GO solution. Finally, we would like also to thank the Microscopy Laboratory of the University of Crete for giving access to the HRTEM microscope.

Conflicts of Interest: The authors declare no conflict of interest.

\section{References}

1. Martín-García, B.; Polovitsyn, A.; Prato, M.; Moreels, I. Efficient charge transfer in solution-processed PbS quantum dot-reduced graphene oxide hybrid materials. J. Mater. Chem. C 2015, 3, 7088-7095. [CrossRef]

2. Fei, H.; Ye, R.; Ye, G.; Gong, Y.; Peng, Z.; Fan, X.; Samuel, E.; Ajayan, P.M.; Tour, J.M. Boron- and Nitrogen-doped graphene quantum dots/graphene hybrid nanoplatelets as efficient electrocatalysts for oxygen reduction. ACS Nano 2014, 8, 10837-10843. [CrossRef] [PubMed]

3. He, J.; Chen, Y.; Li, P.-J.; Fu, F.; Wang, Z.; Zhang, W. Self-assembled $\mathrm{CoS}_{2}$ nanoparticles wrapped by $\mathrm{CoS}_{2}$-quantum-dots-anchored graphene nanosheets as superior-capability anode for lithium-ion batteries. Electrochim. Acta 2015, 182, 424-429. [CrossRef]

4. Gong, C.; Robertson, A.W.; He, K.; Ford, C.; Watt, A.A.R.; Warner, J.H. Interactions of Pb and Te atoms with graphene. Dalton Trans. 2014, 43, 7442-7448. [CrossRef]

5. Zhao, M.; Zhang, J.; Xiao, H.; Hu, T.; Jia, J.; Wu, H.S. Facile in situ synthesis of a carbon quantum dot/graphene heterostructure as an efficient metal-free electrocatalyst for overall water splitting. Chem. Commun. 2019, 55, 1635-1638. [CrossRef]

6. Sygletou, M.; Tzourmpakis, P.; Petridis, C.; Konios, D.; Fotakis, C.; Kymakis, E.; Stratakis, E. Laser induced nucleation of plasmonic nanoparticles on two-dimensional nanosheets for organic photovoltaics. J. Mater. Chem. A 2016, 4, 1020-1027. [CrossRef]

7. Heuer-Jungemann, A.; Kiessling, L.; Stratakis, E.; Kymakis, E.; El-Sagheer, A.H.; Brown, T.; Kanaras, A. Programming the assembly of gold nanoparticles on graphene oxide sheets using DNA. J. Mater. Chem. C 2015, 3, 9379-9384. [CrossRef] 
8. Tan, C.; Cao, X.; Wu, X.-J.; He, Q.; Yang, J.; Zhang, X.; Chen, J.; Zhao, W.; Han, S.; Nam, G.-H.; et al. Recent advances in ultrathin two-dimensional nanomaterials. Chem. Rev. 2017, 117, 6225-6331. [CrossRef]

9. Sygletou, M.; Petridis, C.; Kymakis, E.; Stratakis, E. Advanced photonic processes for photovoltaic and energy storage systems. Adv. Mater. 2017, 29, 1700335. [CrossRef]

10. Busby, Y.; Agresti, A.; Pescetelli, S.; Di Carlo, A.; Noël, C.; Pireaux, J.-J.; Houssiau, L. Aging effects in interface-engineered perovskite solar cells with 2D nanomaterials: A depth profile analysis. Mater. Today Energy 2018, 9, 1-10. [CrossRef]

11. Palma, A.L.; Cinà, L.; Pescetelli, S.; Agresti, A.; Raggio, M.; Paolesse, R.; Bonaccorso, F.; Di Carlo, A. Reduced graphene oxide as efficient and stable hole transporting material in mesoscopic perovskite solar cells. Nano Energy 2016, 22, 349-360. [CrossRef]

12. Attanzio, A.; Rosillo-Lopez, M.; Zampetti, A.; Ierides, I.; Cacialli, F.; Salzmann, C.G.; Palma, M. Assembly of graphene nanoflake-quantum dot hybrids in aqueous solution and their performance in light-harvesting applications. Nanoscale 2018, 10, 19678-19683. [CrossRef] [PubMed]

13. Goodman, A.J.; Dahod, N.S.; Tisdale, W.A. Ultrafast charge transfer at a quantum dot/2D materials interface probed by second harmonic generation. J. Phys. Chem. Lett. 2018, 9, 4227-4232. [CrossRef] [PubMed]

14. Chen, J.-S.; Doane, T.L.; Li, M.; Zang, H.; Maye, M.M.; Cotlet, M. 0D-2D and 1D-2D semiconductor hybrids composed of all inorganic perovskite nanocrystals and single-layer graphene with improved light harvesting. Part. Part. Syst. Charact. 2017, 35, 1700310. [CrossRef]

15. Xu, Y.-F.; Yang, M.-Z.; Chen, B.-X.; Wang, X.; Chen, H.-Y.; Kuang, D.-B.; Su, C.-Y. A CsPbBr 3 perovskite quantum dot/graphene oxide composite for photocatalytic $\mathrm{CO}_{2}$ reduction. J. Am. Chem. Soc. 2017, 139, 5660-5663. [CrossRef] [PubMed]

16. Tang, X.; Zu, Z.; Zang, Z.; Hu, Z.; Hu, W.; Yao, Z.; Chen, W.; Li, S.; Han, S.; Zhou, M. CsPbBr $3 / \operatorname{Reduced}$ Graphene Oxide nanocomposites and their enhanced photoelectric detection application. Sensor Actuat B-Chem 2017, 245, 435-440. [CrossRef]

17. Wang, Q.; Tao, L.; Jiang, X.; Wang, M.; Shen, Y. Graphene oxide wrapped $\mathrm{CH}_{3} \mathrm{NH}_{3} \mathrm{PbBr}_{3}$ perovskite quantum dots hybrid for photoelectrochemical $\mathrm{CO}_{2}$ reduction in organic solvents. Appl. Surf. Sci. 2019, 465, 607-613. [CrossRef]

18. Muduli, S.K.; Pandey, P.; Devatha, G.; Babar, R.; M, T.; Kothari, D.; Kabir, M.; Pillai, P.; Ogale, S. Photoluminescence quenching in self-assembled $\mathrm{CsPbBr} 3$ quantum dots on few-layer black phosphorus sheets. Angew. Chem. Int. Ed. 2018, 57, 7682-7686. [CrossRef]

19. Li, H.; Zheng, X.; Liu, Y.; Zhang, Z.; Jiang, T. Ultrafast interfacial energy transfer and interlayer excitons in the monolayer $\mathrm{WS}_{2} / \mathrm{CsPbBr}_{3}$ quantum dot heterostructure. Nanoscale 2018, 10, 1650-1659. [CrossRef]

20. Akkerman, Q.A.; D'Innocenzo, V.; Accornero, S.; Scarpellini, A.; Petrozza, A.; Prato, M.; Manna, L. Tuning the optical properties of cesium lead halide perovskite nanocrystals by anion exchange reactions. J. Am. Chem. Soc. 2015, 137, 10276-10281. [CrossRef]

21. Kostopoulou, A.; Brintakis, K.; Nasikas, N.K.; Stratakis, E. Perovskite nanocrystals for energy conversion and storage. Nanophotonics 2019, 8, 1607-1640. [CrossRef]

22. Kostopoulou, A.; Kymakis, E.; Stratakis, E. Perovskite nanostructures for photovoltaic and energy storage devices. J. Mater. Chem. A 2018, 6, 9765-9798. [CrossRef]

23. Protesescu, L.; Yakunin, S.; Bodnarchuk, M.I.; Krieg, F.; Caputo, R.; Hendon, C.H.; Yang, R.X.; Walsh, A.; Kovalenko, M.V. Nanocrystals of cesium lead halide perovskites $\left(\mathrm{CsPbX}_{3}, \mathrm{X}=\mathrm{Cl}, \mathrm{Br}\right.$ and I): Novel optoelectronic materials showing bright emission with wide color gamut. Nano Lett. 2015, 15, 3692-3696. [CrossRef] [PubMed]

24. Parobek, D.; Dong, Y.; Qiao, T.; Rossi, D.; Son, D.H. Photoinduced anion exchange in cesium lead halide perovskite nanocrystals. J. Am. Chem. Soc. 2017, 139, 4358-4361. [CrossRef] [PubMed]

25. Sichert, J.A.; Tong, Y.; Mutz, N.; Vollmer, M.; Fischer, S.; Milowska, K.; Cortadella, R.G.; Nickel, B.; Cardenas-Daw, C.; Stolarczyk, J.; et al. Quantum size effect in organometal halide perovskite nanoplatelets. Nano Lett. 2015, 15, 6521-6527. [CrossRef] [PubMed]

26. Bekenstein, Y.; Koscher, B.A.; Eaton, S.W.; Yang, P.; Alivisatos, A.P. Highly luminescent colloidal nanoplates of perovskite cesium lead halide and their oriented assemblies. J. Am. Chem. Soc. 2015, 137, 16008-16011. [CrossRef] 
27. Huang, H.; Li, J.; Yi, Y.; Wang, J.; Kang, Y.; Chu, P.K.; Ong, H.C.; Ong, D.H.C.; Yu, X. In situ growth of all-inorganic perovskite nanocrystals on black phosphorus nanosheets. Chem. Commun. 2018, 54, 2365-2368. [CrossRef]

28. Wu, Y.; Wang, P.; Zhu, X.; Zhang, Q.; Wang, Z.; Liu, Y.; Zou, G.; Dai, Y.; Whangbo, M.-H.; Huang, B. Composite of $\mathrm{CH}_{3} \mathrm{NH}_{3} \mathrm{PbI}_{3}$ with reduced graphene oxide as a highly efficient and stable visible-light photocatalyst for hydrogen evolution in aqueous HI solution. Adv. Mater. 2018, 30, 1704342. [CrossRef]

29. Kostopoulou, A.; Vernardou, D.; Savva, K.; Stratakis, E. All-inorganic lead halide perovskite nanohexagons for high performance air-stable lithium batteries. Nanoscale 2019, 11, 882-889. [CrossRef]

30. Konios, D.; Stylianakis, M.M.; Stratakis, E.; Kymakis, E. Dispersion behavior of graphene oxide and reduced graphene oxide. J. Colloid Interface Sci. 2014, 430, 108-112. [CrossRef]

31. Zhang, Q.; Yin, Y. Strong photoluminescence of $\mathrm{Cs}_{4} \mathrm{PbBr}_{6}$ crystals: a long mystery story. Sci. Bull. 2018, 63, 525-526. [CrossRef]

32. De Weerd, C.; Lin, J.; Gomez, L.; Fujiwara, Y.; Suenaga, K.; Gregorkiewicz, T. Hybridization of single nanocrystals of $\mathrm{Cs}_{4} \mathrm{PbBr}_{6}$ and $\mathrm{CsPbBr}_{3}$. J. Phys. Chem. C 2017, 121, 19490-19496. [CrossRef] [PubMed]

33. Yin, J.; Yang, H.; Song, K.; El-Zohry, A.M.; Han, Y.; Bakr, O.M.; Bredas, J.-L.; Mohammed, O.F. Point defects and green emission in zero-dimensional perovskites. J. Phys. Chem. Lett. 2018, 9, 5490-5495. [CrossRef] [PubMed]

34. Akkerman, Q.A.; Park, S.; Radicchi, E.; Nunzi, F.; Mosconi, E.; De Angelis, F.; Brescia, R.; Rastogi, P.; Prato, M.; Manna, L. Nearly Monodisperse insulator $\mathrm{Cs}_{4} \mathrm{PbX}_{6}(\mathrm{X}=\mathrm{Cl}, \mathrm{Br}$, I) nanocrystals, their mixed halide compositions and their transformation into $\mathrm{CsPbX}_{3}$ nanocrystals. Nano Lett. 2017, 17, 1924-1930. [CrossRef] [PubMed]

35. Ray, A.; Maggioni, D.; Baranov, D.; Dang, Z.; Prato, M.; Akkerman, Q.A.; Goldoni, L.; Caneva, E.; Manna, L.; Abdelhady, A.L. Green-emitting powders of zero-dimensional $\mathrm{Cs}_{4} \mathrm{PbBr}_{6}$ : Delineating the intricacies of the synthesis and the origin of photoluminescence. Chem. Mater. 2019, 31, 7761-7769. [CrossRef]

36. Akkerman, Q.A.; Abdelhady, A.L.; Manna, L. Zero-dimensional cesium lead halides: History, properties and challenges. J. Phys. Chem. Lett. 2018, 9, 2326-2337. [CrossRef]

37. Yang, L.; Li, N.; Wang, C.; Yao, W.; Wang, H.; Huang, K. Room-temperature synthesis of pure perovskite-related $\mathrm{Cs}_{4} \mathrm{PbBr}_{6}$ nanocrystals and their ligand-mediated evolution into highly luminescent $\mathrm{CsPbBr}_{3}$ nanosheets. J. Nanoparticle Res. 2017, 19, 258. [CrossRef]

38. Chen, Y.-M.; Zhou, Y.; Zhao, Q.; Zhang, J.-Y.; Ma, J.-P.; Xuan, T.-T.; Guo, S.-Q.; Yong, Z.-J.; Wang, J.; Kuroiwa, Y.; et al. $\mathrm{Cs}_{4} \mathrm{PbBr}_{6} / \mathrm{CsPBr}_{3}$ perovskite composites with near-unity luminescence quantum yield: Large-scale synthesis, luminescence and formation mechanism and white light-emitting diode application. Acs Appl Mater. Interfaces 2018, 10, 15905-15912. [CrossRef]

39. Liu, Z.; Bekenstein, Y.; Ye, X.; Nguyen, S.C.; Swabeck, J.; Zhang, D.; Lee, S.-T.; Yang, P.; Ma, W.; Alivisatos, A.P. Ligand mediated transformation of cesium lead bromide perovskite nanocrystals to lead depleted $\mathrm{Cs}_{4} \mathrm{PbBr}_{6}$ nanocrystals. J. Am. Chem. Soc. 2017, 139, 5309-5312. [CrossRef]

40. Zhang, D.; Eaton, S.W.; Yu, Y.; Dou, L.; Yang, P. Solution-phase synthesis of cesium lead halide perovskite nanowires. J. Am. Chem. Soc. 2015, 137, 9230-9233. [CrossRef]

41. Kostopoulou, A.; Sygletou, M.; Brintakis, K.; Lappas, A.; Stratakis, E. Low-temperature benchtop-synthesis of all-inorganic perovskite nanowires. Nanoscale 2017, 9, 18202-18207. [CrossRef] [PubMed]

42. Sygletou, M.; Kyriazi, M.-E.; Kanaras, A.; Stratakis, E. Anion exchange in inorganic perovskite nanocrystal polymer composites. Chem. Sci. 2018, 9, 8121-8126. [CrossRef] [PubMed]

43. Alexaki, K.; Kostopoulou, A.; Sygletou, M.; Kenanakis, G.; Stratakis, E. Unveiling the structure of MoS nanocrystals produced upon laser fragmentation of $\mathrm{MoS}_{2}$ platelets. ACS Omega 2018, 3, 16728-16734. [CrossRef] [PubMed]

44. Dang, Z.; Dhanabalan, B.; Castelli, A.; Dhall, R.; Bustillo, K.C.; Marchelli, D.; Spirito, D.; Petralanda, U.; Shamsi, J.; Manna, L.; et al. Temperature-driven transformation of $\mathrm{CsPbBr}_{3}$ nanoplatelets into mosaic nanotiles in solution through self-assembly. Nano Lett. 2020, 20, 1808-1818. [CrossRef]

(C) 2020 by the authors. Licensee MDPI, Basel, Switzerland. This article is an open access article distributed under the terms and conditions of the Creative Commons Attribution (CC BY) license (http://creativecommons.org/licenses/by/4.0/). 